\title{
Graph-Based Optimization with Tubularity Markov Tree for 3D Vessel Segmentation
}

\author{
Ning Zhu and Albert C.S. CHUNG \\ Lo Kwee-Seong Medical Image Analysis Laboratory, \\ Department of Computer Science and Engineering, \\ The Hong Kong University of Science and Technology, Hong Kong \\ nzhu@cse.ust.hk, achung@cse.ust.hk
}

\begin{abstract}
In this paper, we propose a graph-based method for $3 D$ vessel tree structure segmentation based on a new tubularity Markov tree model (TMT), which works as both new energy function and graph construction method. With the help of power-watershed implementation [7], a global optimal segmentation can be obtained with low computational cost. Different with other graph-based vessel segmentation methods, the proposed method does not depend on any skeleton and ROI extraction method. The classical issues of the graph-based methods, such as shrinking bias and sensitivity to seed point location, can be solved with the proposed method thanks to vessel data fidelity obtained with TMT. The proposed method is compared with some classical graph-based image segmentation methods and two up-to-date $3 D$ vessel segmentation methods, and is demonstrated to be more accurate than these methods for $3 D$ vessel tree segmentation. Although the segmentation is done without ROI extraction, the computational cost for the proposed method is low (within 20 seconds for $256 * 256 * 144$ image).
\end{abstract}

\section{Introduction}

In recent years, graph-based methods, such as graph cuts [4, 5], random walker [9], power-watershed [7] and their extensions [6, 10, 11, 17, 22, 23], have become a group of popular image segmentation methods. These graph-based methods can be used to obtain global optimal segmentation from computationally efficient energy optimization. A unifying framework for graph-based energy optimization methods for two-class image segmentation is proposed in [7]. The objective functions of graph cuts, random walker, power-watershed then can be seen as special cases of the framework by employing particular parameters.

Vessel disease, which is one of the major causes of death around the world, has become an important health problem. Vessel segmentation is very useful for the diagnosis, visualization, treatment and surgery planning of vessel diseases. With the advanced development of the medical image acquisition modalities, such as CT and MRI, many vessel segmentation methods have been developed in recent years $[12,14,16,18,19,21]$. However some classical segmentation methods cannot achieve good performance on some specific medical applications, such as vessel segmentation. Here are some challenges for 3D vessel segmentation: a) The segmentation of elongated structures of vessels may suffer from the shrinking bias (Graph Cuts) and sensitivity of seed point locations (Random walker). These two problems are easy to solve in $2 \mathrm{D}$ by providing more seed points. However, for 3D images, especially for vessel structures which spread over a large area in the image, providing enough seed points is not an easy task. b) As can be seen in Figures 1(a) and 1(b), vessels may be in or near some other organs. This makes the segmentation more difficult. c) The intensity can change significantly along a vessel branch. So the intensity feature which is widely used in many segmentation methods is not a good choice for vessel segmentation. Also, the distal part of some vessels may be obscure (Figure 1(c)), and this may cause segmentation leakage to the background area. As a result, despite of the advantages of graph-based framework, only a few methods [3, 8, 17, 20, 24], which combine graph cuts with vessel shape priors, are used for vessel segmentation. Graph cuts based methods in $[17,20,24]$ are used to obtain global optimal segmentation for single branches. However, for the whole vessel tree segmentation, the simple combination of different branches may not be sufficient. Methods in [3, 8] first detect the skeleton of vessel tree and then the graph cuts method is used for whole tree optimization. All these graph cuts based methods require obtaining vessel skeleton as preprocessing and the region of interest (ROI) are then extracted accordingly. As a result, the performance of these 


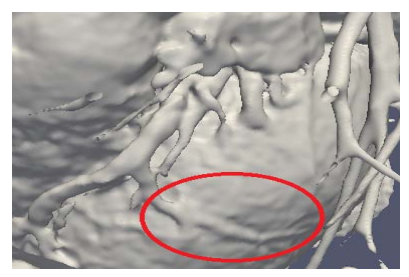

(a) Coronary artery on heart

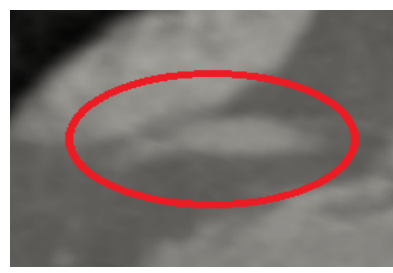

(b) Vessel near other object

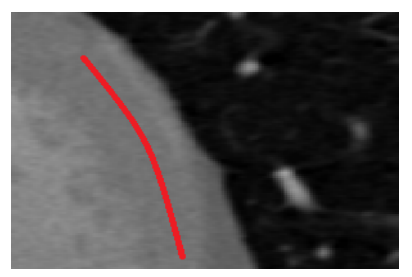

(c) Distal part of vessel

Figure 1. Difficulties for 3D Vessel Segmentation

methods rely heavily on the accuracy of skeleton detection. Paper Contributions. In this paper, we propose a new graph-based method for vessel tree structure segmentation based on a new tubularity Markov tree model (TMT), which works as both new energy function and graph construction method. The proposed method has four favorable properties: (i) With the new TMT model, a good data fidelity is embedded in the new objective function (Equation 2) and is evaluated to be effective on increasing the accuracy and reducing the risk of shrinking bias. (ii) The optimization is done on the whole 3D image without extracting the ROI. As a result, the performance of the proposed method does not depend on the skeleton extraction results. (iii) The optimal segmentation result can be obtained with low computation cost. And (iv), the bifurcation of vessel tree structure does not require particular consideration.

\section{Background}

In this section, some background definitions will be provided. For graph-based methods, graph construction and energy function are two major factors affecting the segmentation performance. Here we define the undirected graph as $G^{o}=\left(V^{o}, E^{o}\right)$ with node set $V^{o}$ and edge set $E^{o} . v_{i}$ and $e_{i j}$ are nodes and edges in $V^{o}$ and $E^{o}$ respectively. $w_{i j}$ is the weight for edge $e_{i j}$. In this paper, we assume $G$ is a undirected graph, so here we have $w_{i j}=w_{j i}$. In [7], the authors proposed a unifying framework for graph optimization methods for two-class segmentation:

$$
\begin{aligned}
& \text { Step } 1: \mathbf{x}= \arg \min _{x} \sum_{e_{i j} \in E^{o}} w_{i j}^{p}\left|x_{i}-x_{j}\right|^{q} \\
&+\sum_{v_{i} \in V^{o}} w_{F_{i}}^{p}\left|x_{i}\right|^{q}+\sum_{v_{i} \in V^{o}} w_{B_{i}}^{p}\left|x_{i}-1\right|^{q} \\
& \text { s.t. } x(F)=1, x(B)=0, \\
& \text { Step 2: } s_{i}=1 \text { if } x_{i} \geq \frac{1}{2}, 0 \text { if } x_{i}<\frac{1}{2}
\end{aligned}
$$

Here $x_{i}$ and $s_{i}$ are the foreground probability and result label for node $v_{i}$ respectively. $\mathbf{x}$ is the probability set for all the nodes in $V^{o}$. As discussed in [7], when $p$ is a small finite number, the optimization for Equation 1 can be done with graph cuts $(q=1)$ or random walker $(q=2)$. And for $p \rightarrow \infty$, the objective function can be optimized with power watershed method proposed in [7] when $q$ is a finite number. This framework (Equation 1) actually summarizes all the energy based graph optimization methods for two-class object segmentation.

\section{Proposed Method}

In this section, the proposed method will be described in two parts. First, a new framework will be created for segmenting a particular class from multiple objects background. Then TMT based new energy function and graph construction method for the objective function (Equation 2) will be introduced.

\subsection{Singular Object Segmentation Objective}

As described in Section 2, the framework (Equation 1) is designed for two-class object segmentation. As can be seen in Figure 1(b), some blood vessels, e.g., the coronary arteries, are surrounded with different objects, such as heart, vertebra, tissue, and other types of vessels. However, coronary artery is the only target we want to segment. Generally speaking, in many applications, we may need to separate a particular type of object out of a complex environment. And the segmentation task for 3D images is especially important and more difficult than that for 2D images. For vessel tree segmentation, we modify the unifying framework (Equation 1) from [7] to the following objective function:

$$
\begin{aligned}
& \mathbf{x}=\arg \min _{x} \lim _{p \rightarrow \infty}\left(\sum_{e_{i j} \in E^{o}} w_{i j}^{p}\left|x_{i}-x_{j}\right|^{2}+\sum_{v_{i} \in V_{t m t}} T_{w_{i}}^{p}\left|x_{i}-1\right|^{2}\right) \\
& \text { s.t. } x\left(S_{V}\right)=1, x\left(S_{B}\right)=0 .
\end{aligned}
$$

Here $T M T=\left(E_{t m t}, V_{t m t}\right)$ is a tubularity Markov tree structure with its edge set $E_{t m t}$ and node set $V_{t m t}$, and $T_{w_{i}}$ is the tubularity Markov response for $v_{i} \in V_{t m t} . S_{V}$ and $S_{B}$ are the vessel seeds and background seeds. In this objective function, we delete the original region energy terms for both foreground and background and add a new data fidelity term for the nodes in the set $V_{t m t}$. This is because we are trying to segment the object out from multiple types 
of background objects and it is difficult to model the region energy for foreground and background with single models. Usually, the energy term is based on voxel intensity distribution. However, it is impossible to identify the vessel voxel from the others with only intensity since the background body organs may have similar intensity. We will introduce the details of the new data fidelity term in Section. 3.2.

In this objective function, we use $p \rightarrow \infty$ and $q=2$ in the unifying framework. $p$ here represents to what extent the weights are emphasized. For 3D vessel segmentation, $p \rightarrow \infty$ is the best choice since this can reduce the risk of shrinking bias, and this is especially important for elongated structure segmentation with small number of seed points.

\subsection{Tubularity Markov Tree and Graph Construc- tion}

In this section, we propose a new graph-based optimization method for vessel segmentation. A new energy function based on tubularity Markov tree model is proposed and used as vessel fidelity, and accordingly a new graph construction method is described.

To construct a good vessel data fidelity, the tubularity Markov tree model is proposed. In this model, we make use of the tubularity shape character of vessel structure with a Markov tree representation. The method for tubularity Markov tree construction is described in Algorithm 1. The red lines in Figure 2 sketches the structure for the tubularity Markov tree. The red tree in the figure is just a main structure showing the construction of TMT. In real application, the tree may be much more dense, especially in the area within and near the vessels. And the neighboring relation of nodes is voxel-based. So different with that shown in the figure, there may be many nodes on the path between the root and the nodes outside the vessels. Given a start seed node, the tubularity Markov tree is constructed by adding the nodes with the largest weighted joint tubularity score (shortened as tubularity score, $T_{s}$ ) iteratively. The implementation of TMT construction is similar with the classic Prim's minimal spanning tree in the manner of nodes spanning. One of the major differences is that the Prim's algorithm needs to reach all nodes in the graph. But in the proposed method, the spanning will be stopped if there is no candidate with high score $\left(T_{s} \geq \tau\right)$ in the stack. And also, the spanning of the Prim's tree actually can be seen as the first order Markov process for adding nodes with the least edge cost connecting with the spanning tree. While in the proposed method, we aim at adding the nodes with the largest weighted joint tubularity score $\left(T_{s}\right)$ into the tree. The tubularity score is calculated according to the following

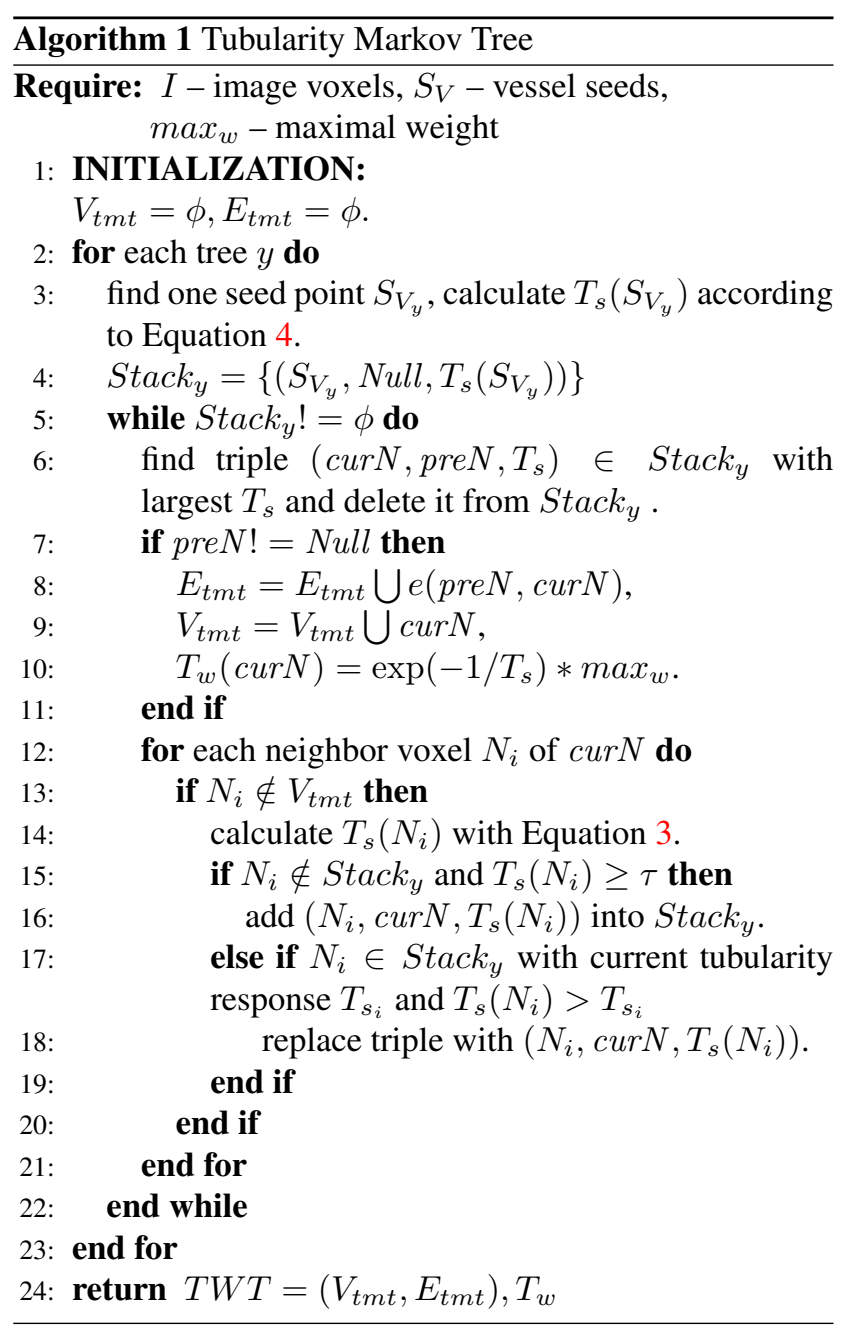

equation when $i>1$ :

$$
\begin{aligned}
& T_{s}\left(v_{i}\right)=\lambda * T_{s}\left(v_{i-1}\right)+(1-\lambda) * m f\left(v_{i}, d_{i}\right) \\
& =\lambda^{i-1} * T_{s}\left(v_{1}\right)+(1-\lambda) * \sum_{x=2}^{i} \lambda^{i-x} * m f\left(v_{x}, d_{i}\right),
\end{aligned}
$$

where $d_{i}=v_{i}-v_{i-1}$. When $i=1$, we assume

$$
T_{s}\left(v_{1}\right)=\max _{d_{1}^{\prime}} m f\left(v_{1}, d_{1}^{\prime}\right)
$$

in which $d_{1}^{\prime}$ represents the directions from $v_{1}$ to its neighbors. Here we use $v_{i-1}$ to represent the parent node for $v_{i}$ in the tree. Mflux is used in [13, 15] as the medialness feature for centerline tracking. Based on Mflux, we define a Max-Mflux response as:

$$
\begin{aligned}
& m f\left(p_{t}, d\right)=\max _{r}\left\{\frac{2}{N} \sum_{i=1}^{\frac{N}{2}} \min \left(\left(\nabla \boldsymbol{I}\left(a_{i}\right) \cdot \boldsymbol{u}_{i}\right),\left(\nabla \boldsymbol{I}\left(a_{i}^{\pi}\right) \cdot \boldsymbol{u}_{i}^{\pi}\right)\right)\right\} \\
& \boldsymbol{u}_{i}=\left(p_{t}-a_{i}\right) /\left|p_{t}-a_{i}\right|, \quad a_{i}^{\pi}=a_{\frac{2}{N}+i} .
\end{aligned}
$$




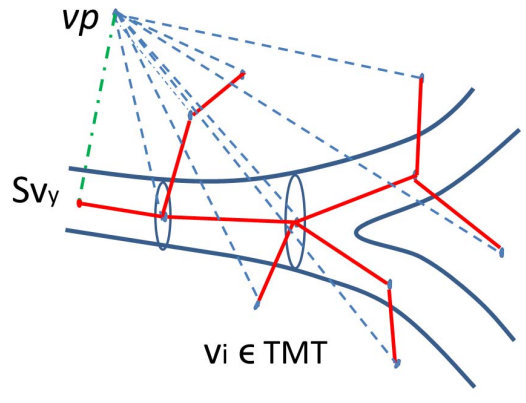

Figure 2. TMT Graph

Given $p_{t}$ as the center point and $r$ as radius, $a_{i}$ is point on the circle. $\nabla \boldsymbol{I}\left(a_{i}\right)$ is the gradient vector of $a_{i}$, and the included angle between $p_{t}-a_{i}$ and $p_{t}-a_{i+1}$ for $i=1, \ldots, N-1$ are $2 \pi / N$. Different with Mflux, MaxMflux search all the possible $r$ and return the largest Mflux response. In [15], Mflux is seen as the response for a node being candidate on the centerline. In this paper, we construct a Markov tree with the tubularity score $T_{s}$ based on Max-Mflux. For each node, all the ancestor nodes' MaxMflux responses are considered with degradation weights and the weights are summed to 1 (Equation 3). This is the reason that we can make use of the changed medialness feature as the response of the node being candidate in the vessel. For the nodes in the vessel but deviate from the center, it may have a series of high Max-Mflux response ancestors. As a result, although the response for the nodes itself may not be high, the weighted joint tubularity score considering all the ancestors may not be low. And for the points with high Max-Mflux response for both the ancestors and itself, it can have a high tubularity score and as a result has a high data fidelity as foreground vessel point. The new tubularity score is robust for the seed node position. Take a perfect tube for example, the Max-Mflux response is the same on centerline no matter where the seed point is located. Since the weights in $T_{s}$ is summed to 1 , the tubularity scores for all centerline points are the same, regardless whatever $\lambda$ is. Then the spanning may reach the nodes near all centerline points with the same ancestor's score. The parameter $\lambda$ can be used to control the spanning range. Given threshold $\tau$ fixed, if $\lambda$ is large, the impact of the ancestors is emphasized, and the spanning can go further thanks to ancestors' good performance. Otherwise if $\lambda$ is small, the node needs to depend on itself and if it does not have good Max-Mflux, the spanning will not cover this node.

The proposed new model is named as tubularity Markov tree model, here the "tree" represents the spanning structure, and the relationship between a particular node and its ancestors is more likely a Markov chain. The spanning of the tree is based on the new weighted joint tubularity score. All these together with the graph construction accomplish the proposed TMT model.

After obtaining the tree and tubularity response, the graph is constructed according to Algorithm 2, which is the main framework for the proposed method. $v p$ is a virtual graph node for implementation. $e\left(v p, V_{t m t}\right)$ here represents the edge set including all the edges connecting the node $v p$ and nodes in $V_{t m t}$. These edges are represented by blue dashed lines in Figure 2. Similarly, $e\left(v p, S_{v}\right)$ represents all the edges connecting $v p$ and the vessel seed points. Green dashed in Figure 2 line gives one example in this edge set. The graph constructed with Algorithm 2 includes two parts, the image lattices $\left(G^{o}\right)$ and the TMT part $\left(G^{\prime}\right)$, which is sketched in Figure 2 (dash lines and associate nodes). After the graph construction, the power-watershed [7] method is used to optimize the objective function (Equation 2). Here we give a proof illustrating that the objective function (Equation 2) can be optimized by Algorithm 2.

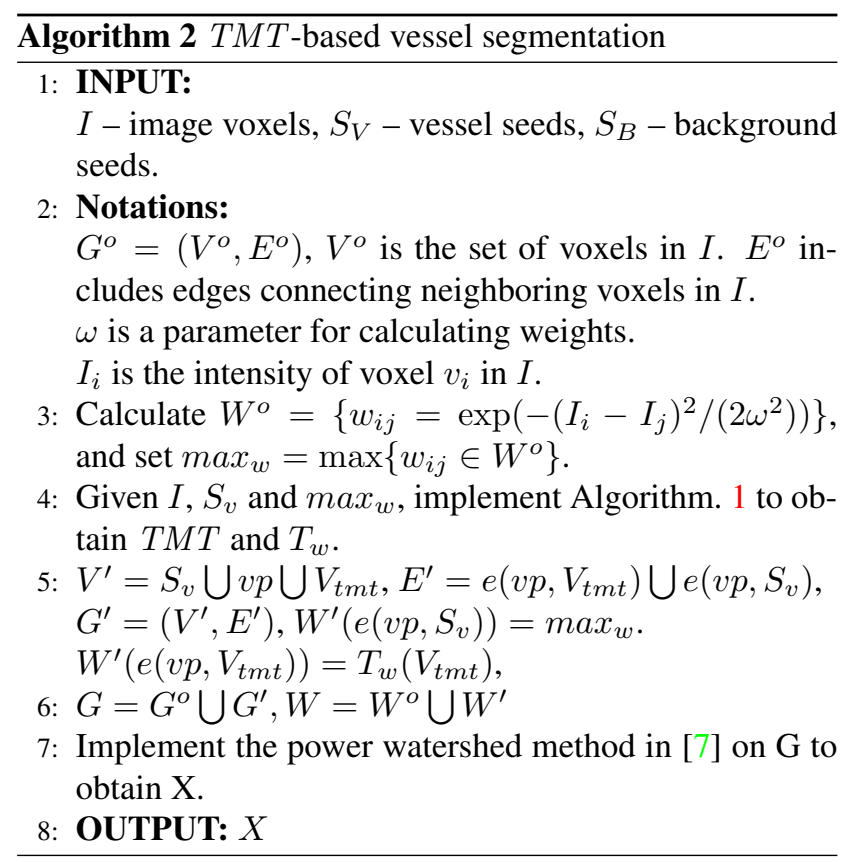

Proof: Since $x\left(S_{v}\right)=1$, we have

$$
\sum_{v_{i} \in V_{t m t}} T_{w_{i}}^{p}\left|x_{i}-1\right|^{2}=\sum_{v_{i} \in V_{t m t}} T_{w_{i}}^{p}\left|x_{i}-x\left(S_{v}\right)\right|^{2} .
$$

According to the implementation of power watershed method [7], since $W^{\prime}\left(e\left(v p, S_{v}\right)\right)=\max _{w}, v p$ and all the seeds in $S_{v}$ will be merged to one node first, then we have $x\left(S_{v}\right)=x(v p)$. Let $E^{\prime \prime}$ be the sets of edges $e\left(v p, V_{t m t}\right)$, and $W^{\prime \prime}\left(e\left(v p, V_{t m t}\right)\right)=T_{w}\left(V_{t m t}\right)$ be the weights of $E^{\prime \prime}$, then we have,

$$
\sum_{v_{i} \in V_{t m t}} T_{w_{i}}^{p}\left|x_{i}-1\right|^{2}=\sum_{e_{i j} \in E^{\prime \prime}} w_{i j}^{p}\left|x_{i}-x_{j}\right|^{2} .
$$

And $E=E^{\prime \prime} \cup E^{o}$ after the merging $v p$ and $S_{v}$, so finally, 


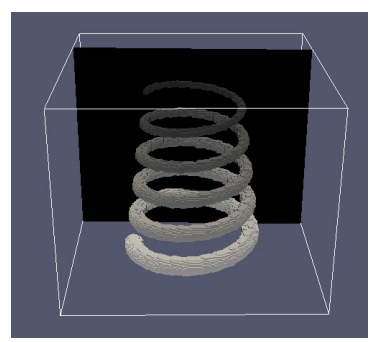

(a) Synthetic Image 1

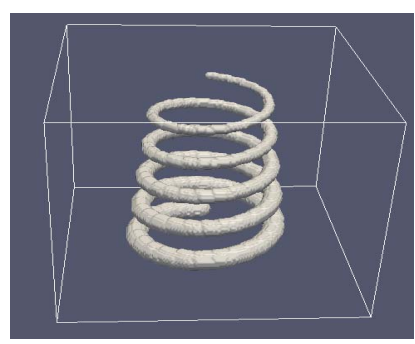

(b) proposed method (sigma $=100)$

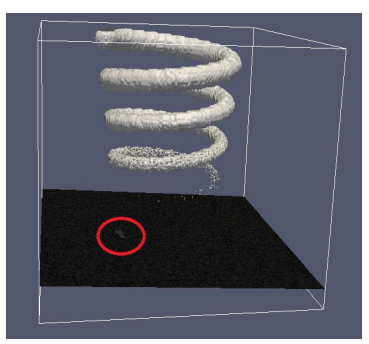

(c) Paraview $($ sigma $=100)$

Figure 3. Experiments on Synthetic Image 1

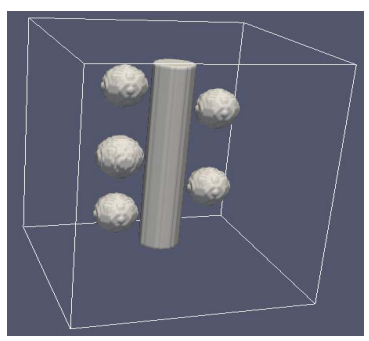

(a) Synthetic Image 2

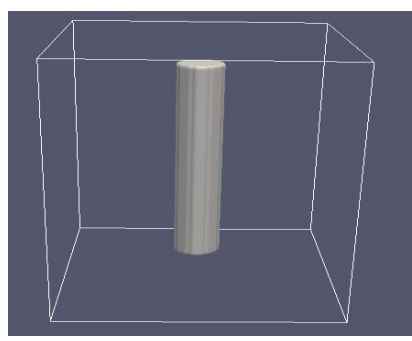

(b) proposed method(sigma $=200$ )

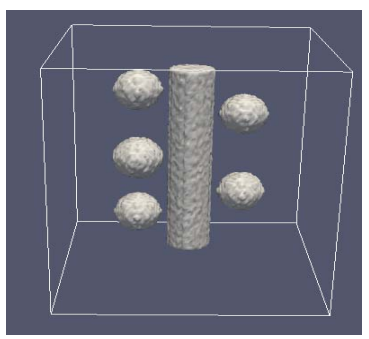

(c) Paraview $($ sigma $=200)$

Figure 4. Experiments on Synthetic Image 2

the objective function (Equation 2) is same with the following one after first step node merging.

$$
\begin{aligned}
& x=\arg \min _{x} \lim _{p \rightarrow \infty} \sum_{e_{i j} \in E} w_{i j}^{p}\left|x_{i}-x_{j}\right|^{2} \\
& \text { s.t. } x\left(S_{V}\right)=1, x\left(S_{B}\right)=0,
\end{aligned}
$$

It is proved that this objective function can be optimized with power watershed method in [7]. As a result, the original objective function (Equation 2) can be optimized with the proposed method presented in Algorithm 2.

In this section, a new model named TMT is proposed, and new data fidelity and graph construction method based on the model is also introduced. Based on the new weighted joint tubularity score in Equation 3, and the tree spanning method described in Algorithm 1, the TMT model provides good data fidelity, and perform preliminary graph construction. Algorithm 2 is then used to construct the whole graph for energy optimization of the new singular object segmentation objective function (Equation 2).

\section{Experiments}

For method evaluation, we have compared the proposed method with some other segmentation methods on both synthetic images and clinical CTA images. First, an group of experiments have been carried out on synthetic images (Figure 3(a) and Figure 4(a)). As stated in Section 1, two important challenges for vessel segmentation are: a) the ves- sels are surrounded by human organs with similar intensity (Figure 1(b)), b) the distal part of vessel may be obscure (Figure 1(c)). In the synthetic experiments, the images are designed to simulate these two problem and different methods are evaluated on these images. The first synthetic image is shown in Figure 3(a). The intensity of the tubular structure ranges from 1200 to 850 , and the background intensity is set to 800. Similar with real vessels, the radius of the synthetic vessel is also decreased gradually. The size of the image is $200 * 200 * 150$ voxels. In the second synthetic image (Figure 4(a)), the intensity of synthetic vessel and nearby objects are set to 1200 and that of background is set to 800 . The smallest distance between the objects and the tube ranges from 1-3 voxels, and the size of this image is $100 * 100 * 100$ voxels. Gaussian noise was added to the synthetic images to generate a series of noisy synthetic images for the evaluation.

The proposed method was compared with powerwatershed method [7], graph cuts [4] on the two groups of synthetic images, and to be fair, we also conduct the comparison with the graph cuts method by providing the same graph constructed with the proposed method. Here a widely used segmentation measure DICE is employed for evaluating the accuracy of segmentation results. As can be seen in Table 1, the segmentation accuracy of the proposed method is good even for highly noisy image. However, the accuracy of the other three methods drops dramatically down. Synthetic image 1 is generated with the intensity of its distal 
Table 1. DICE and Computation Time on Synthetic Image 1

\begin{tabular}{|c|cccccc|}
\hline$\sigma$ (noise level) & WithoutNoise & 20 & 40 & 60 & 100 & Time(Seconds) \\
\hline Our method & $100 \%$ & $100 \%$ & $99.9996 \%$ & $98.4314 \%$ & $97.6359 \%$ & 17.931 \\
\hline Power WaterShed [7] & $100 \%$ & $99.9996 \%$ & $99.9982 \%$ & $97.6285 \%$ & $4.5408 \%$ & 11.472 \\
\hline GraphCut [4] & $100 \%$ & $100 \%$ & $4.5762 \%$ & $4.5762 \%$ & $4.5762 \%$ & 29.523 \\
\hline GraphCut With TMT & $100 \%$ & $100 \%$ & $100 \%$ & $4.5762 \%$ & $4.5762 \%$ & 31.742 \\
\hline
\end{tabular}

Table 2. DICE on Synthetic Image 2

\begin{tabular}{|c|cccc|}
\hline$\sigma$ (noise level) & WithoutNoise & 100 & 200 & 400 \\
\hline Our method & $100 \%$ & $95.9341 \%$ & $88.1162 \%$ & $70.2263 \%$ \\
\hline Power WaterShed [7] & $100 \%$ & $96.0211 \%$ & $6.2724 \%$ & $6.4620 \%$ \\
\hline GraphCut [4] & $100 \%$ & $100 \%$ & $6.3913 \%$ & $6.3913 \%$ \\
\hline GraphCut With TMT & $100 \%$ & $95.9341 \%$ & $83.7718 \%$ & $6.3913 \%$ \\
\hline
\end{tabular}

part dark and when the noise level increases, the leakage to the background for both graph cut methods and watershed method may happen. The segmentation result then shrink to the background seeds points and all the other voxels in the image are labeled as foreground object. The results for synthetic image 2 are shown in Table 2. All methods performs perfectly on the image without noise. However, when the noise level increases, the background results of the other three methods again shrink to the background seed points. The accuracy of the graph cuts method with TMT graph drops later than the other two methods, which means with $T M T$, a better performance on noisy images is obtained for the graph cuts method. This may be a indicator of the good performance for TMT graph. By providing reasonable data fidelity which can reduce the shrinking risk, the proposed method can have good performance on noisy images. Different with the graph cuts method with $T M T$, our method emphasizes more on the weights by $p \rightarrow \infty$ and does not suffer from the shrinking bias.

In all experiments, the weight $\lambda$ for calculating the tubularity score was set to 0.5. While $\omega$ in Algorithm 2 was set to 200 in the synthetic experiments and 20 for the real images because this parameter is closely related to boundary intensity change. $\tau$ in Algorithm 1 was set to 80 for synthetic images and 40 for real images. $r$ in Equation. 5 ranges from $0.6 \mathrm{~mm}$ to $4.8 \mathrm{~mm}$.

The average computation time was recorded for synthetic image 1 and listed in Table 1. The experiments are carried out on a server with 4xAMD Opteron $844(1.8 \mathrm{GHz})$ CPU and 8GB RAM. With the proposed method, the segmentation can be finished within 18 seconds on average for the synthetic image with size $200 * 200 * 150$ voxels. The 18 seconds includes the TMT construction time (about 8 seconds) and optimization time. The TMT construction is the reason that the proposed methods and the Graph Cuts method with TMT require more the computation time than watershed and graph cuts method respectively. Actually, although it takes a few seconds to obtain TMT and the graph can be a little larger than that in watershed and graph cuts method, the optimization procedure for the proposed method and graph cuts with TMT may be accelerated with the help of TMT construction.

Figures 3(b) and 4(b) show the surface obtained with the proposed method for noisy image with $\sigma=100$ and $\sigma=200$. Since the obtained surface of the other methods shrinks to points, here we present the contours obtained with Paraview software [1] as a supplement. Figures 3(c) and 4(c) presents the contours obtained with Paraview [1] software on the noisy image with $\sigma=100$ and $\sigma=200$. In Figure 3(c), a slice of distal part of the synthetic vessel is also presented. As shown in the figure, Paraview does well in the parts with high contrast and large vascular radius, while it cannot track the distal parts with low contrast and high noise level. And in Figure 4(c), since there is no seed point given to Paraview, it finds all the objects while in the proposed method, we aim at finding the vascular structure. The surface obtained with the proposed method is more smoother than the ones obtained with Paraview.

The experiments was also carried out on clinical images for coronary artery segmentation. As can be seen in Figure 5, the proposed method is compared with classic region growing [2] (Figure 5(a)), graph cuts [4] (Figure 5(b)), graph cuts with TMT graph (Figure 5(c)) and power watershed method [7] (Figure 5(d)) on a 256*256*144 clinical image. As shown in these figures, region growing and graph cuts methods suffer a lot from leakage since the distal parts of the vessel are obscure and have no clear boundary. Given a seed point (red point in Figure 5(a)), the region growing method grows to the whole heart area and also spread to some other vessels nearby. And the tracking of real objective coronary artery (green part in Figure 5(a)) stops earlier so the distal parts are missing. Since the distal parts of the real vessels are not clear, the obtained results of graph cuts methods leak to other organs and vessels near the coronary arteries. As for the power watershed method, although it emphasizes more on weights which may reduce the risk of shrinking, the unpredictable intensity changes along the vessels makes it difficult to track the distal parts. As a result, it encounters with the problem of shrinkage. For the proposed method, with the vessel fidelity from $T M T$, the 


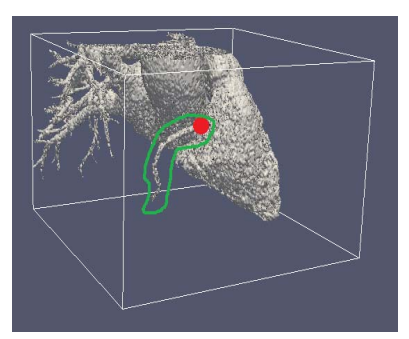

(a) Region Growing

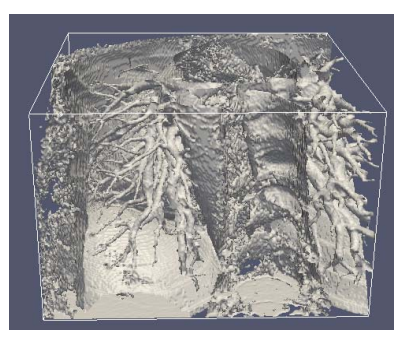

(b) Graph Cuts [4]

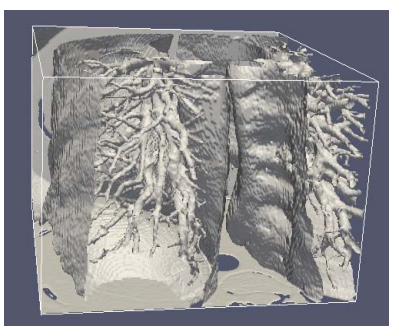

(c) Graph Cuts with $T M T$

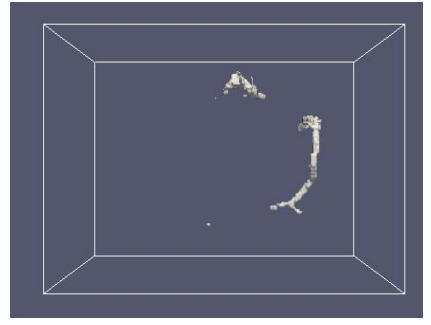

(d) Power Watershed[7]

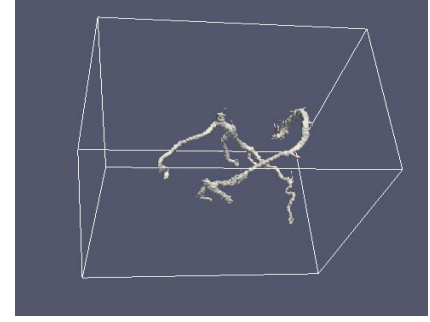

(e) Proposed Method

Figure 5. Segmentation Results on Clinical Image 1

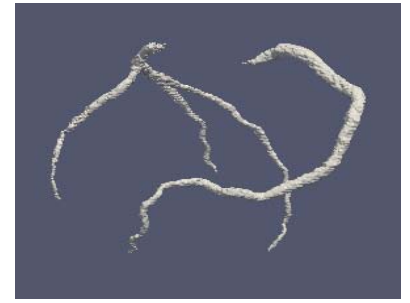

(a) Proposed method

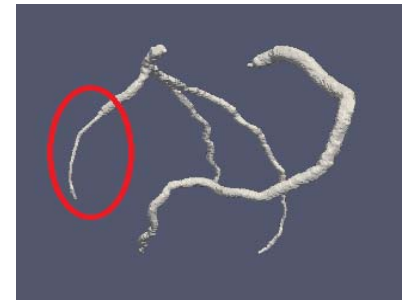

(b) Surface obtained with [3]

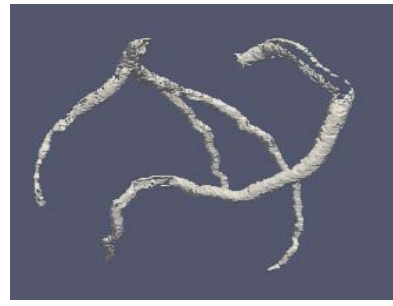

(c) Surface obtained with[8]

Figure 6. Segmentation Results on Clinical Image 2

segmentation does not suffer from either shrinking bias or leakage. The computation time of the proposed method in this experiment is 19.384 seconds.

The proposed method was also compared with two graph-based vessel segmentation methods [8, 3]. In these two methods, the skeletons are first detected and the graph cuts methods are applied on the region of interest. So in this group of experiments, we first detect the centerline of the vessels with the method in [25] and find the region of interest according to the associated radius obtained. With the same ROI, and seed points, the segmentation results of the three methods are shown in Figure 6. As shown in the figure, the surfaces for [3] seem smoother. However, without the help of regional energy, the method in [3] suffers from shrinking problem, which makes some distal parts thinner than the real segmentation. The segmentation results in this group seem better than the the results in Figure 5(e). However, they require the skeleton of the vessels as premise. As a result, the performance of these methods depends on the accuracy of the skeleton detection for the vessel structures. Given a good estimation of skeleton, these methods will be good choices. While in the cases that skeletons are not obtainable or not accurate, methods that do not require accurate skeleton are needed. The proposed method, as may be noticed in these experiments, can adapt in both situations. Without ROI, it works well for obtaining the objective vessels; while given accurate skeleton, it performs better since the neighboring objects with same intensity are eliminated.

\section{Conclusion}

In this paper, we propose a graph-based method for 3D vessel tree structure segmentation based on a new tubularity Markov tree model (TMT), which works as both new energy function and graph construction method. With the help of power-watershed implementation, a global optimal segmentation can be obtained with low computational cost. Different with all other graph-based vessel segmen- 
tation methods, the proposed method does not depend on any skeleton and ROI extraction method. The classical issues of the graph-based method, such as shrinking bias and sensitivity to seed point position, can be solved with the proposed method thanks to the TMT model as vessel data fidelity in the graph-based unifying framework. The proposed method is compared with some classical graph-based image segmentation methods and two up-to-date 3D vessel segmentation methods. The experiments show that the proposed method is more accurate than these methods for $3 \mathrm{D}$ vessel tree segmentation. The computational time for the whole tree segmentation is within 20 seconds although the whole 3D image is included in the algorithm.

We would like to acknowledge the financial support of the Hong Kong Research Grants Council under grant 612011.

\section{References}

[1] Paraview, http://www.paraview.org/. 6

[2] R. Adams and L. Bischof. Seeded region growing. Pattern Analysis and Machine Intelligence, IEEE Transactions on, 16(6):641-647, 1994. 6

[3] C. Bauer, T. Pock, and et al. Segmentation of interwoven 3d tubular tree structures utilizing shape priors and graph cuts. Medical Image Analysis, 14(2):172-184, 2010. 1, 7

[4] Y. Boykov and G. Funka-Lea. Graph cuts and efficient nd image segmentation. International Journal of Computer Vision, 70(2):109-131, 2006. 1, 5, 6, 7

[5] Y. Boykov and M. Jolly. Interactive graph cuts for optimal boundary \& region segmentation of objects in nd images. In Eighth IEEE International Conference on Computer VisionICCV, volume 1, pages 105-112, 2001. 1

[6] Y. Boykov and V. Kolmogorov. An experimental comparison of min-cut/max-flow algorithms for energy minimization in vision. IEEE Transactions on Pattern Analysis and Machine Intelligence, 26(9):1124-1137, 2004. 1

[7] C. Couprie, L. Grady, L. Najman, and H. Talbot. Power watershed: a unifying graph-based optimization framework. Pattern Analysis and Machine Intelligence, IEEE Transactions on, 33(7):1384-1399, 2011. 1, 2, 4, 5, 6, 7

[8] S. Esneault, C. Lafon, and et al. Liver vessels segmentation using a hybrid geometrical moments/graph cuts method. IEEE Transactions on Biomedical Engineering, 57(2):276283, 2010. 1, 7

[9] L. Grady. Random walks for image segmentation. Pattern Analysis and Machine Intelligence, IEEE Transactions on, 28(11):1768-1783, 2006. 1

[10] L. Grady and M. Jolly. Weights and topology: A study of the effects of graph construction on 3d image segmentation. In Medical Image Computing and Computer-Assisted Intervention-MICCAI, pages 153-161, 2008. 1

[11] S. Hanaoka, K. Fritscher, M. Welk, M. Nemoto, Y. Masutani, N. Hayashi, K. Ohtomo, and R. Schubert. 3-d graph cut segmentation with riemannian metrics to avoid the shrinking problem. Medical Image Computing and Computer-Assisted Intervention-MICCAI, pages 554-561, 2011. 1
[12] C. Kirbas and F. Quek. A review of vessel extraction techniques and algorithms. ACM Computing Surveys, 36(2):81121, 2004. 1

[13] D. Lesage, E. Angelini, I. Bloch, and G. Funka-Lea. Design and study of flux-based features for $3 \mathrm{~d}$ vascular tracking. In Biomedical Imaging: From Nano to Macro, 2009. ISBI'09. IEEE International Symposium on, pages 286-289. IEEE, 2009. 3

[14] D. Lesage, E. Angelini, and et al. A review of 3D vessel lumen segmentation techniques: Models, features and extraction schemes. Medical Image Analysis, 13(6):819-845, 2009. 1

[15] D. Lesage, E. Angelini, and et al. Bayesian maximal paths for coronary artery segmentation from 3D CT angiograms. In Medical Image Computing and ComputerAssisted Intervention-MICCAI, pages 222-229, 2009. 3, 4

[16] H. Li and A. Yezzi. Vessels as 4-D curves: Global minimal 4D paths to extract 3-D tubular surfaces and centerlines. IEEE Transactions on Medical Imaging, 26(9):1213, 2007. 1

[17] K. Li, X. Wu, and et al. Optimal surface segmentation in volumetric images-a graph-theoretic approach. IEEE Transactions on Pattern Analysis and Machine Intelligence, 28(1):119-134, 2006. 1

[18] L. Lorigo, O. Faugeras, W. Grimson, R. Keriven, R. Kikinis, A. Nabavi, and C. Westin. Curves: Curve evolution for vessel segmentation. Medical Image Analysis, 5(3):195-206, 2001. 1

[19] F. Quek and C. Kirbas. Vessel extraction in medical images by wave-propagation and traceback. IEEE Transactions on Medical Imaging, 20(2):117-131, 2001. 1

[20] M. Schaap, L. Neefjes, and et al. Coronary lumen segmentation using graph cuts and robust kernel regression. In Information Processing in Medical Imaging, pages 528-539, 2009. 1

[21] M. Schaap, T. van Walsum, L. Neefjes, C. Metz, E. Capuano, M. de Bruijne, and W. Niessen. Robust shape regression for supervised vessel segmentation and its application to coronary segmentation in cta. Medical Imaging, IEEE Transactions on, 30(11):1974-1986, 2011. 1

[22] D. Singaraju, L. Grady, and et al. P-brush: Continuous valued mrfs with normed pairwise distributions for image segmentation. In IEEE Conference on Computer Vision and Pattern Recognition-CVPR, pages 1303-1310, 2009. 1

[23] S. Vicente, V. Kolmogorov, and C. Rother. Graph cut based image segmentation with connectivity priors. In IEEE Conference on Computer Vision and Pattern Recognition-CVPR, pages 1-8, 2008. 1

[24] F. Zhao, H. Zhang, and et al. Congenital aortic disease: 4d magnetic resonance segmentation and quantitative analysis. Medical Image Analysis, 13(3):483-493, 2009. 1

[25] N. Zhu and A. Chung. Minimum average-cost path for real time $3 \mathrm{~d}$ coronary artery segmentation of ct images. In Medical Image Computing and Computer-Assisted InterventionMICCAI, 2011. 7 